\title{
Simultaneous measurements of terahertz emission and magneto-optical Kerr effect for resolving ultrafast laser-induced demagnetization dynamics
}

\author{
T. J. Huisman, ${ }^{1}$ R. V. Mikhaylovskiy, ${ }^{1}$ A. Tsukamoto, ${ }^{2}$ Th. Rasing, ${ }^{1}$ and A. V. Kimel ${ }^{1}$ \\ ${ }^{1}$ Radboud University Nijmegen, Institute for Molecules and Materials, 6525 AJ Nijmegen, Netherlands \\ ${ }^{2}$ College of Science and Technology, Nihon University, 7-24-1 Funabashi, Chiba, Japan
}

(Received 17 December 2014; published 17 September 2015)

\begin{abstract}
Simultaneous detection of terahertz $(\mathrm{THz})$ emission and transient magneto-optical response is employed to study ultrafast laser-induced magnetization dynamics in three different types of amorphous metallic alloys: Co, GdFeCo, and NdFeCo. A satisfactory agreement between the dynamics revealed with the help of these two techniques is obtained for $\mathrm{Co}$ and $\mathrm{GdFeCo}$. For NdFeCo the $\mathrm{THz}$ emission indicates faster dynamics than the magneto-optical response. This observation indicates that in addition to spin dynamics of $\mathrm{Fe}$, ultrafast laser excitation of $\mathrm{NdFeCo}$ triggers faster magnetization dynamics of $\mathrm{Nd}$ originating from its orbital momentum.
\end{abstract}

DOI: 10.1103/PhysRevB.92.104419

PACS number(s): 75.78.Jp, 78.30.Er

\section{INTRODUCTION}

The seminal observation of subpicosecond demagnetization in ferromagnetic nickel by a laser pulse published almost two decades ago [1] triggered the field of ultrafast magnetization dynamics - a topic that has been continuously fueled by intriguing observations as well as controversies in the scientific community [2-8]. One of the main reasons for the controversies is the lack of an artifact-free technique capable of observing the magnetization dynamics at the subpicosecond time scale. Most experimental studies of ultrafast magnetism performed so far employ an all-optical pump-probe technique in which the magnetization is probed indirectly via the magneto-optical Faraday or Kerr effect. However, it was noticed that at the subpicosecond time scale the magneto-optical probes are subject to artifacts [9]. In particular, it was argued that if the temporal behavior of the Kerr ellipticity is different from the one of the Kerr rotation, the dynamics of the magnetooptical signal cannot be directly associated with the true magnetization dynamics [10]. However, the opposite statement is not obviously true and similar behavior of the ellipticity and the rotation cannot be used as a proof that the dynamics of the magneto-optical Kerr effect (MOKE) adequately reflects the magnetization dynamics. Additional complications in the interpretation of time-resolved magneto-optical experiments arise from element sensitivity of magneto-optical effects and the presence of both spin and orbital magnetization, especially in multisublattice magnets [11-16]. Alternatively, in order to deduce information about subpicosecond dynamics of the total magnetization one can employ the fact that such dynamics is accompanied by the emission of terahertz (THz) electromagnetic radiation [17-20]. To discover the potential use of $\mathrm{THz}$ radiation for the study of ultrafast magnetism, we performed simultaneous measurements of the MOKE and the electric field of the emitted $\mathrm{THz}$ radiation.

We show that nonlinear optical effects of nonmagnetic origin or the inverse spin Hall effect cannot be responsible for the observed emission of the $\mathrm{THz}$ radiation, and thus the emission originates from the ultrafast demagnetization. Under assumption that the MOKE represents the dynamics of the net magnetization, we deduce the spectrum of the $\mathrm{THz}$ emission which is generated by such magnetization dynamics by solving the Maxwell equations for a magnetic film, taking into account the propagation of the $\mathrm{THz}$ radiation from the emitter to the detector. Comparing these calculated spectra with the actually measured ones we reveal that there is a very good match between the spectra for a pure Co film. This observation verifies the assumptions underlying the calculations. Using the same parameters for the spectrometer response, we demonstrate that while qualitative similarities between the spectra are obtained for $\mathrm{GdFeCo}$ alloys, in $\mathrm{NdFeCo}$ alloys there are clear discrepancies between the $\mathrm{THz}$ spectra expected from the MOKE dynamics and the actually measured $\mathrm{THz}$ spectra. From considering several explanations, we speculate that the discrepancy stems from the orbital magnetism of $\mathrm{Nd}$ playing a role in the demagnetization process.

\section{EXPERIMENTAL METHODS}

Our experimental approach uniquely combines $\mathrm{THz}$ timedomain spectroscopy (THz-TDS) with an optical pump-probe scheme, as sketched in Fig. 1(a). An amplified titaniumsapphire laser system is used, producing light pulses with a duration of $50 \mathrm{fs}$ at a repetition rate of $1 \mathrm{kHz}$ and with a center wavelength of $800 \mathrm{~nm}$. The laser beam is divided into three parts: pump, probe, and gate. The pump is focused onto an area on the sample with a diameter of approximately $1 \mathrm{~mm}$. The pump fluence is approximately $1 \mathrm{~mJ} / \mathrm{cm}^{2}$. THz radiation emitted from the sample is collected and focused onto a 1 $\mathrm{mm}$ thick ZnTe crystal using two parabolic mirrors. The $\mathrm{THz}$ radiation induces birefringence inside the $\mathrm{ZnTe}$ crystal due to the electro-optic Pockels effect. By probing this birefringence using the gate pulse and a balanced bridge detection scheme, we are able to reconstruct the electric field of the emitted $\mathrm{THz}$ radiation as a function of time. Simultaneously, the probe pulse is focused on the sample to a spot which is approximately two times smaller in diameter than the pump with a fluence of approximately ten times less than the pump. The angle of incidence of the probe beam is $25^{\circ}$. By measuring the polarization rotation of the reflected probe pulse, information about the ultrafast laser-induced magnetization dynamics is obtained by means of the MOKE. The measurements have been performed in magnetic fields up to $1 \mathrm{kG}$ applied in-plane of the samples. Performing the measurements for two polarities of the magnetic field and taking the difference between the measurements, one can deduce signals odd with respect to 

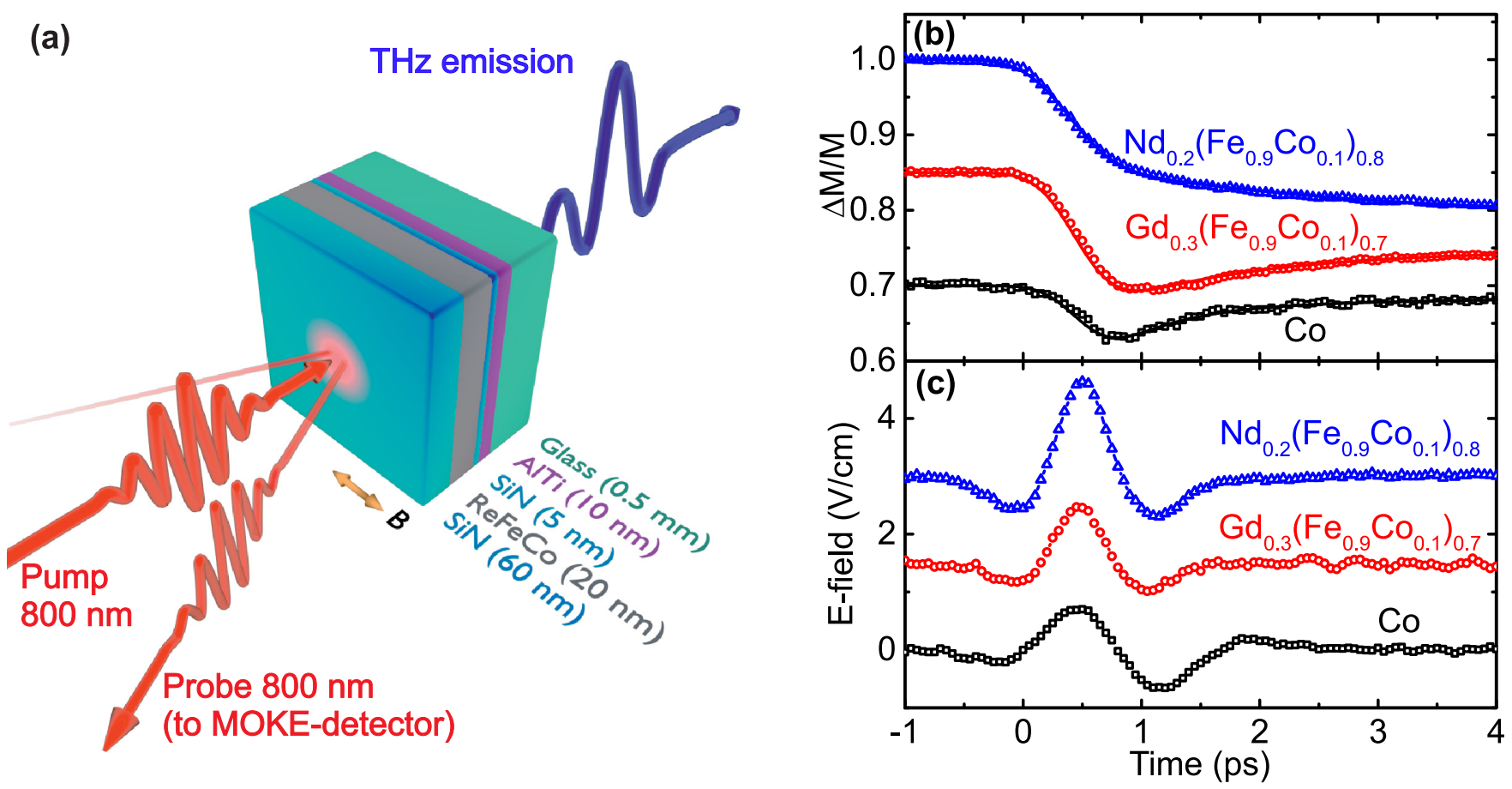

FIG. 1. (Color online) (a) The layered structure under study and the scheme of the experiment. Re is rare earth, being either Nd or Gd. The reflected probe pulse is used to measure the MOKE signal. The electric field of the THz emission is detected with the help of electro-optic (EO) detection. A field of $\pm 1 \mathrm{kG}$ is applied in plane. (b) Ultrafast magnetization dynamics as deduced from time-resolved MOKE measurements. The solid lines are fitting functions as expressed in Eqs. (10) and (11). (c) Electric field of the emitted THz radiation as a function of time. The position of zero time delay is chosen arbitrarily so that the position of the peak of the pump pulse is close to zero. The traces are vertically shifted with respect to each other.

the field and thus minimize any influence of artifacts of nonmagnetic origin. Simultaneous MOKE and THz detection of the laser-induced dynamics minimizes potential ambiguities, which may arise when comparing two different experiments. We note that despite the uniqueness of combining $\mathrm{THz}$ emission and the MOKE techniques, this approach is generally accessible. Practically every pump-probe setup measuring ultrafast dynamics triggered by femtosecond laser pulses can be easily combined with the detection of $\mathrm{THz}$ emission.

Both probes of ultrafast demagnetization are applied to Co, GdFeCo, and NdFeCo alloys. A 12-nm-thick ferromagnetic Co film is deposited on a $0.5-\mathrm{mm}$-thick glass substrate. Such a sample is a typical example of a Stoner ferromagnet used in many ultrafast magnetization dynamics studies and therefore acts as a starting reference point. The multisublattice magnetic materials studied in this work are rare-earth transition-metal alloys. The results presented on these materials are mainly for $\mathrm{Nd}_{0.2}\left(\mathrm{Fe}_{0.87} \mathrm{Co}_{0.13}\right)_{0.8}$ and $\mathrm{Gd}_{0.3}\left(\mathrm{Fe}_{0.87} \mathrm{Co}_{0.13}\right)_{0.7}$, measured at room temperature. We note that temperature-dependent measurements in the range $150-300 \mathrm{~K}$ as well as similar measurements on $\mathrm{Nd}_{0.5}\left(\mathrm{Fe}_{0.87} \mathrm{Co}_{0.13}\right)_{0.5}$ and $\mathrm{Gd}_{0.18}\left(\mathrm{Fe}_{0.87} \mathrm{Co}_{0.13}\right)_{0.82}$ show similar results. The GdFeCo alloys are ferrimagnetic and the magnetic properties of these alloys are essentially determined by the fact that the Gd spin moments ( $4 f$ and $5 d$ ) are aligned oppositely to the spin moments of $\mathrm{Fe}$ and $\mathrm{Co}(3 d)$. Importantly, in $\mathrm{Gd}_{0.18}\left(\mathrm{Fe}_{0.87} \mathrm{Co}_{0.13}\right)_{0.82}$ the FeCo magnetization is larger than the one of $\mathrm{Gd}$ at all temperatures, while in $\mathrm{Gd}_{0.3}\left(\mathrm{Fe}_{0.87} \mathrm{Co}_{0.13}\right)_{0.7}$ the net magnetization is dominated by the Gd sublattice. Since the MOKE probe is mainly sensitive to the magnetization of the FeCo sublattice while the $\mathrm{THz}$ radiation is expected to be emitted from the net magnetization change, the different probing techniques are expected to show different results at least in sign for the two GdFeCo alloys studied. Furthermore the orbital momenta of $\mathrm{Fe}$ and $\mathrm{Gd}$ in the GdFeCo alloys are expected to be quenched, while for $\mathrm{Nd}$ the magnetization is dominantly determined by its orbital momentum. In the NdFeCo alloys, the orbital momentum of $\mathrm{Nd}$ is larger than the spin of $\mathrm{Nd}$ and aligned antiparallel with respect to it. Hence, despite the antiferromagnetic coupling of the Fe and $\mathrm{Nd}$ spins, the alloy is effectively ferromagnetic. The NdFeCo and GdFeCo alloys were incorporated into a layered structure with different layers to prevent oxidation (capping layer $\mathrm{SiN}$ ), reduce laser-induced heating (heat sink AlTi), and facilitate the growth (buffer layer $\mathrm{SiN}$ ). The layered structure is shown in Fig. 1(a). Our work therefore includes three different classes of magnetic ordering: ferromagnetic spin ordering without (significant) orbital momentum (Co), and ferrimagnetic spin ordering without $(\mathrm{GdFeCo})$ and with orbital momentum ( $\mathrm{NdFeCo})$. All the magnetic films have in-plane magnetic anisotropy with coercive fields below $150 \mathrm{G}$ at room temperature.

\section{EXPERIMENTAL RESULTS}

Figure 1(b) shows the results of all-optical pump-probe experiments in which the laser-induced dynamics is detected with the help of the MOKE. The transient signals were calibrated using static MOKE measurements. The figure shows 



FIG. 2. (Color online) (a) Electric field of the emitted THz radiation from the $\mathrm{Nd}_{0.2}\left(\mathrm{Fe}_{0.87} \mathrm{Co}_{0.13}\right)_{0.8}$ sample as a function of time. The measurements have been performed in an external magnetic field of $1 \mathrm{kG}$ and $-1 \mathrm{kG}(+\mathrm{B}$ and $-\mathrm{B})$. The wave forms are measured for two orientations of the sample as indicated next to the curves. The inset shows the hysteresis of the peak amplitude when the pump pulse excites the sample from the side of the substrate. (b) MOKE measured demagnetization for $\mathrm{Gd}_{0.18}\left(\mathrm{Fe}_{0.87} \mathrm{Co}_{0.13}\right)_{0.82}$ and $\mathrm{Gd}_{0.3}\left(\mathrm{Fe}_{0.87} \mathrm{Co}_{0.13}\right)_{0.7} . \mathrm{The}$ solid lines are fitting functions as expressed in Eqs. (10) and (11). (c) Measured demagnetization emission of $\mathrm{Gd}_{0.18}\left(\mathrm{Fe}_{0.87} \mathrm{Co}_{0.13}\right)_{0.82}$ and $\mathrm{Gd}_{0.3}\left(\mathrm{Fe}_{0.87} \mathrm{Co}_{0.13}\right)_{0.7}$. The traces are vertically shifted with respect to each other. The position of zero time is chosen arbitrarily so that the position of the peak of the pump pulse is close to zero.

the data in units of the relative change of the magnetization. $\mathrm{NdFeCo}$ shows clearly distinct dynamics from that of $\mathrm{GdFeCo}$ and the reference Co sample. These deviating dynamics can be explained by a different response of the various materials to the optical pump.

Figure 1(c) shows the temporal evolution of the electric field of the emitted radiation for the three samples. With the help of wire-grid polarizers, we found that the electric field of the $\mathrm{THz}$ emission from all the samples is linearly polarized, perpendicular to the magnetization. Figure 2(a) shows the THz wave forms measured for the $\mathrm{Nd}_{0.2}\left(\mathrm{Fe}_{0.87} \mathrm{Co}_{0.13}\right)_{0.8}$ alloy, which is representative for all the studied samples. The traces clearly change sign when the applied field is reversed. Moreover, measuring the peak amplitude as a function of the applied magnetic field reveals a hysteresis behavior as shown in the inset of Fig. 2(a). This observation is a clear demonstration of the fact that the electric field of the $\mathrm{THz}$ emission is proportional to the magnetization of the magnetic layer.

This magnetization dependence likely also means that our emission is of magnetic dipole origin, i.e., a rapid quenching of the magnetization which causes emission. However, it can be argued that laser excitation of a free-electron gas in a magnetic field could also result in $\mathrm{THz}$ emission, which is of electric dipole origin [21]. Such an electric dipole emission is allowed only in noncentrosymmetric media and can be especially efficient at the interfaces of the magnetic film. One of the microscopic realizations of this mechanism is suggested in Ref. [20] showing that the $\mathrm{THz}$ emission is generated due to the inverse spin Hall effect experienced by a spin-polarized current from a magnetic to a nonmagnetic layer. To check for emission generated by such a spin current from the magnetic layer, we inverted the sample by turning it around the magnetic field axis over $180^{\circ}$ while keeping the applied magnetic field fixed. In this way the direction of a potential spin current between adjacent layers has to reverse sign. Therefore the sign of the $\mathrm{THz}$ radiation which would originate from this current should be reversed as well. Figure 2(a) shows the $\mathrm{THz}$ wave forms after turning the sample around. Besides different pulse widths and amplitude changes, which are mostly due to absorption in the glass substrate, no sign change is observed. The measurements on all other samples showed similar results. Hence, the experiments have revealed no indication that the emitted $\mathrm{THz}$ radiation is due to a spin current or similar symmetry-breaking effects. From all these features we conclude that ultrafast laser-induced demagnetization can be reliably assigned to be the main source of the observed $\mathrm{THz}$ emission from the studied samples.

As we noted above for the GdFeCo alloys, the MOKE probe is expected to be sensitive to the magnetization of the $\mathrm{FeCo}$ sublattice, while the $\mathrm{THz}$ emission comes from the net magnetization change. The two studied $\mathrm{GdFeCo}$ alloys, $\mathrm{Gd}_{0.18}\left(\mathrm{Fe}_{0.87} \mathrm{Co}_{0.13}\right)_{0.82}$ and $\mathrm{Gd}_{0.3}\left(\mathrm{Fe}_{0.87} \mathrm{Co}_{0.13}\right)_{0.7}$, have different ratios between the magnetizations of the sublattices. For $\mathrm{Gd}_{0.18}\left(\mathrm{Fe}_{0.87} \mathrm{Co}_{0.13}\right)_{0.82}$ the magnetization of the FeCo sublattice dominates the net magnetization, while for $\mathrm{Gd}_{0.3}\left(\mathrm{Fe}_{0.87} \mathrm{Co}_{0.13}\right)_{0.7}$ the magnetization of the $\mathrm{Gd}$ sublattice dominates the net magnetization. Figure 2(b) shows the dynamics of the MOKE measured for these two samples. It is seen that the dynamics of these two alloys have opposite signs, which is the result of different sublattices 
(a)

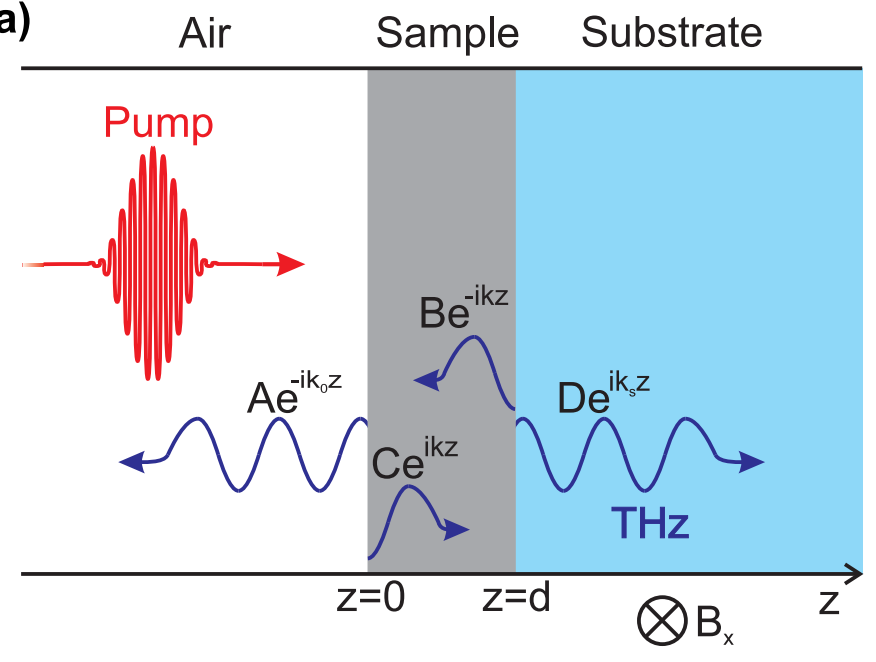

(b)

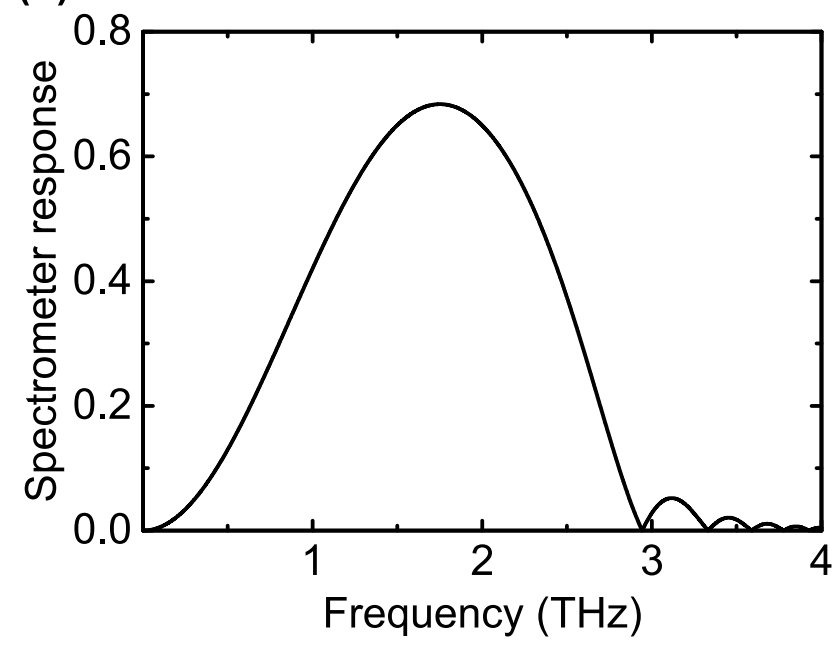

FIG. 3. (Color online) (a) A laser pump pulse initiates magnetization dynamics in the sample which is accompanied by the emission of electromagnetic radiation. (b) Response of the spectrometer as a function of frequency.

dominating the magnetization. Figure 2(c) shows that the signs of the $\mathrm{THz}$ emission are the same for the two GdFeCo samples. The difference in the amplitudes of the emitted $\mathrm{THz}$ radiation is expected to be related to the difference in the net magnetizations.

\section{ANALYSIS METHOD}

In previous studies, the ultrafast demagnetization emission and magneto-optical probe data were compared with each other assuming that the laser-induced magnetization dynamics acts as a magnetic point-dipole emitter [17]. Such an approach disregards any effect of optical components on the propagation of the radiation and significant size of the source. As a result, it may lead to an unreliable estimate of the spectral distribution and amplitude of the emission. To avoid these uncertainties, we solve the Maxwell equations with corresponding boundary conditions for an infinite film. The calculations are performed for a thin metal film in the $x y$ plane on top of a substrate as indicated in Fig. 3(a). The film is assumed to be magnetized along the $x$ axis and exhibits homogeneous magnetization dynamics. From Ampere's and Faraday's laws we can relate a current source to the generated electromagnetic radiation (in Gaussian units):

$$
\frac{\partial^{2} \tilde{E}_{y}(z)}{\partial z^{2}}+\frac{\omega^{2}}{c^{2}} \varepsilon(z) \tilde{E}_{y}(z)=-\frac{4 \pi i \omega}{c^{2}} \tilde{J}_{y}(z),
$$

where $\tilde{E}_{y}$ is the $y$ component of the electric field, $\varepsilon(z)$ is the dielectric permittivity, $\omega$ is the angular frequency, $c$ is the speed of light, and $\tilde{J}_{y}$ is the $y$ component of the current density; the $\sim$ symbol is used to indicate the Fourier transform with respect to time.

We are looking for the solutions in the form of the fundamental solution of the homogeneous wave equation [see Fig. 3(a)], from which follows

$$
\tilde{E}_{y}(z)= \begin{cases}A e^{-i k_{0} z}, & z<0 \\ B e^{-i k z}+C e^{i k z}, & 0<z<l, \\ D e^{i k_{s} z}, & z>L\end{cases}
$$

with $k_{0}$ being the wave vector in vacuum or air, $k$ is the wave vector in the metallic layers, and $k_{s}$ is the wave vector in the substrate. From Faraday's law we know that the electric field of Eq. (2) at the interfaces should be continuous. The current reads $\tilde{J}_{y}=c \frac{\partial \tilde{M}_{x}(z)}{\partial z}=c \tilde{m}_{x}[\delta(z+d)-\delta(z)]$, with $\delta(z)$ being the Dirac delta function and $d$ the thickness of the magnetic layer. From this and Eq. (1), we can recognize that the derivative of the electric field at the left interface of Fig. 3(a) exhibits a discontinuity of $\varphi=-\frac{4 \pi i \omega \tilde{m}_{x}}{c}$, and a similar discontinuity by an amount of $-\varphi$ at the right interface of Fig. 3(a). This provides the boundary conditions,

$$
\left\{\begin{array}{l}
A=B+C, \quad z=0 \\
-k_{0} A=-k B+k C-\phi / i, \quad z=0 \\
B e^{-i k d}+C e^{i k d}=D e^{i k_{s} d}, \quad z=d \\
-k B e^{-i k d}+k C e^{i k d}=k_{s} D e^{i k_{s} d}+\phi / i, \quad z=d .
\end{array}\right.
$$

Solving these equations for $D$, i.e., the emitted electric field amplitude, provides us with

$$
\begin{aligned}
D e^{i k_{s} d}= & \frac{4 \pi \omega}{c} \tilde{m}_{x} \\
& \times \frac{\left(k-k_{0}\right) e^{i k d}+\left(k+k_{0}\right) e^{-i k d}-2 k}{\left(k+k_{0}\right)\left(k+k_{s}\right) e^{-i k d}-\left(k-k_{0}\right)\left(k-k_{s}\right) e^{i k d}} .
\end{aligned}
$$

Since we are working with thin films, we can approximate Eq. (4) using a Taylor expansion of the form $e^{ \pm i k d} \approx 1 \pm i k d$. Assuming that the metal films have a significant conductivity such that $k^{2} \gg k_{0} k_{s}$, we simplify Eq. (4) as

$$
D e^{i k_{s} d} \approx-\frac{4 \pi \omega}{c} \tilde{m}_{x} \frac{i d}{(1+n)+4 \pi \sigma d / c},
$$

with $n$ being the refractive index of the substrate and $\sigma$ the conductivity of the metallic layer. For high conductivity and thick metallic layers [thick layer limit, i.e., $(1+n) \ll$ $4 \pi \sigma d / c]$ we retrieve the emitted electric field being

$$
\tilde{E}_{y} \approx-\frac{i \omega}{\sigma} \tilde{m}_{x} e^{i \frac{\omega}{c} n z}
$$


On the other hand, for low conductivity and thin metallic layers [thin layer limit, i.e., $(1+n) \gg 4 \pi \sigma d / c$ ] we can see that

$$
\tilde{E}_{y} \approx-\frac{4 \pi i \omega}{c} \tilde{m}_{x} \frac{d}{1+n} e^{i \frac{\omega}{c} n z} .
$$

Both Eqs. (6) and (7) provide the same frequency dependence, as the conductivity in the spectral range of interest can be taken as constant [22].

The observed spectrum of the emitted electromagnetic radiation can be related to the spectrum of the generated radiation by a linear relation,

$$
E_{\text {observed }}=K(\omega) E_{\text {source }},
$$

where $K(\omega)$ is the transfer function which accounts for the substrate transmission, propagation effects, and response of the $\mathrm{ZnTe}$ crystal. If the thickness of the substrate is much larger than the wavelength of the electromagnetic wave, the transmission through such a substrate is given by the Fresnell transmission equation,

$$
t=\frac{2 n_{i}}{n_{i}+n_{t}},
$$

where $n_{i}$ is the refractive index of the medium from which the radiation originates and $n_{t}$ is the refractive index of the medium to which the radiation is transmitted. The refractive index of the glass substrate is obtained experimentally with the help of $\mathrm{THz}$ transmission spectroscopy as described in [23]. Our results are comparable with the observations in Ref. [24]; the absorption of glass can be approximated by a quadratic function which increases upon an increase of the frequency of the $\mathrm{THz}$ radiation. Due to this fact, glass effectively suppresses high frequencies of the emitted radiation.

To come from a near-field solution to the $\mathrm{THz}$ radiation at the detecting crystal, we model Gaussian propagation similar to the one described in [25]. In order to model Gaussian propagation, one needs to provide an initial diameter for the Gaussian beam in the model. Our initial diameter used in the calculations is taken equal to the diameter of the pump beam at the sample. In our setup, the effects of propagation remove low frequencies in the spectrum. Frequencies above $2 \mathrm{THz}$ are all enhanced by a factor equal to the ratio of the focal lengths of the two parabolic mirrors used to collect and refocus the $\mathrm{THz}$ emission. For modelling the $\mathrm{ZnTe}$ response we applied the methods mentioned in [26], which shows that higher frequencies are suppressed. To compare quantitatively the calculated spectra with the experimentally obtained ones, we used Eq. (9) from [27] which shows how the observed ellipticity in the ZnTe crystal is related to the electric field of the $\mathrm{THz}$ radiation. Taking into account both the propagation effects and the ZnTe response defines the spectrometer response, which can be visualized as a bandpass filter centered around 1.7 THz; see Fig. 3(b). The parameters required to calculate this spectrometer response are taken from the geometry of our setup. The ZnTe crystal response attenuates frequencies above $1.7 \mathrm{THz}$, while effects of propagation attenuate frequencies below $1.7 \mathrm{THz}$. While the MOKE measurements themselves would be easily able to reach a spectral sensitivity up to $10 \mathrm{THz}$, our spectrometer response for $\mathrm{THz}$ radiation limits the spectral sensitivity to up to $3 \mathrm{THz}$, or up to $2 \mathrm{THz}$ when taking into account absorption in the glass substrate. However, already in a spectral range from 0 to $2 \mathrm{THz}$, comparing MOKE and $\mathrm{THz}$ emission may provide more insight on their relative functioning and reveal discrepancies.

\section{DISCUSSION}

To determine the generated electromagnetic radiation from the MOKE probe, the spectrum $\tilde{m}_{x}$ is required. To prevent numerical errors, the observed MOKE rotation is fitted with an empirical function, which is either

$$
\frac{\Delta M}{M}=\left\{\frac{1}{2} \operatorname{erf}[F(t-G)]+\frac{1}{2}\right\}\left(H e^{-I t}+J e^{-K t}\right),
$$

or

$$
\frac{\Delta M}{M}=\left\{\frac{1}{2} \operatorname{erf}[F(t-G)]+\frac{1}{2}\right\}\left(H \ln (I t) e^{-J t}\right),
$$

with $F, G, H, I, J$, and $K$ as fitting parameters. The choice of the fitting function depends on the shape of MOKE rotation in time. Figure 1(b) shows the magneto-optical probe data fitted using Eqs. (10) and (11). Figure 4(a) shows the electromagnetic radiation emitted as a result of the magnetization dynamics inferred from the time-resolved MOKE data using only Eq. (7).

In Fig. 4(b) we compare our measured spectra (solid lines) with spectra calculated from the time-resolved MOKE data (dashed lines) for different samples. For the calculated spectrum of Co we assumed that the net magnetization is equal to $1400 \mathrm{emu} / \mathrm{cm}^{3}$ [28]. For $\mathrm{Gd}_{0.3}\left(\mathrm{Fe}_{0.87} \mathrm{Co}_{0.13}\right)_{0.7}$ we took the magnetization equal to $150 \mathrm{emu} / \mathrm{cm}^{3}$ [29]. For $\mathrm{Nd}_{0.2}\left(\mathrm{Fe}_{0.87} \mathrm{Co}_{0.13}\right)_{0.8}$ we used $462 \mathrm{emu} / \mathrm{cm}^{3}$ as measured with the help of a vibrating magnetometer and consistent with the literature [30-32]. The narrower spectra obtained for Co compared to the other samples is the result of using a thicker glass substrate. This thicker substrate results in more absorption of higher-frequency radiation [24]. The spectra obtained for the Co sample show excellent mutual agreement. These observations show that $\mathrm{THz}$ emission and MOKE for a pure ferromagnetic material provide identical information of the ultrafast magnetization dynamics and verify experimentally our calculated spectrometer response. This spectrometer response is kept identical for all samples.

For GdFeCo the spectrum calculated from the MOKE and the actually measured spectrum of the $\mathrm{THz}$ emission show a mismatch in amplitudes by a factor of 2. Simultaneously, we observe that the full width at half maximum (FWHM) of the spectrum obtained using the MOKE agrees well with the FWHM of the actually measured THz spectrum (see Table I). The expectation values of the spectra are also similar. Hence there is a satisfactory quantitative and a good qualitative agreement between the spectrum calculated from the MOKE and the actually measured spectrum of the $\mathrm{THz}$ emission during ultrafast laser-induced demagnetization of GdFeCo.

On the other hand, for the case of ultrafast laser-induced demagnetization of $\mathrm{NdFeCo}$, no qualitative agreement between the spectrum calculated from the MOKE and the actually measured spectrum of the $\mathrm{THz}$ emission was obtained. In particular, Fig. 4(b) clearly indicates the lack 



FIG. 4. (Color online) (a) The calculated spectra of the emitted electromagnetic radiation inferred from the time-resolved magneto-optical data shown in Fig. 1(b), using Eq. (7). (b) The detected spectra of the THz emission. The solid lines (1) are spectra obtained with the help of the Fourier transform of the measured THz wave forms. The dashed lines (2) indicate the calculated spectra of (a) and taking into account the spectrometer response for $\mathrm{THz}$ radiation. The calculated spectra of GdFeCo and NdFeCo are shown multiplied by 2 and 1.8 , respectively. The shaded areas are meant to highlight the difference between the calculated and measured spectra.

of higher frequencies in the spectrum calculated from the MOKE response of NdFeCo; see also Table I. This implies that the dynamics revealed by the MOKE is slower than the magnetization dynamics revealed with the help of $\mathrm{THz}$ emission spectroscopy.

To determine the origin of the observed disagreements in the spectra, we note that the GdFeCo and $\mathrm{NdFeCo}$ samples have similar structures. Hence the layered structure of the studied samples cannot explain the qualitatively different results obtained for these two types of alloys. Also as we discuss in the Appendix, this discrepancy does not come from the fact that we performed our calculations in the thin layer limit, using Eq. (7).

One may argue that the observed discrepancies between the calculated and measured spectra for $\mathrm{NdFeCo}$ can be due to elemental specificity of the MOKE, similar to what we observed for our GdFeCo alloys. If the dynamics measured with the MOKE in NdFeCo is associated with the spin dynamics of the FeCo sublattice, as expected for an 800-nm-wavelength probe, the mismatch in spectral distribution shows the presence of faster magnetization dynamics which remains invisible for the MOKE detection. This is surprising as x-ray measurements revealed that for $\mathrm{GdFeCo}$ alloys, the Gd spin sublattice demagnetizes slower than the spins of FeCo [13]. Analyzing the similarities and differences of $\mathrm{GdFeCo}$ and $\mathrm{NdFeCo}$ alloys indicates that the faster dynamics in $\mathrm{NdFeCo}$ must originate from the presence of orbital magnetization of $\mathrm{Nd}$. As the discrepancies are mostly apparent above $1 \mathrm{THz}$, this indicates that the orbital dynamics occur on a time scale faster than 1 ps. Resolving the exact dynamics of spin momentum, orbital momentum, and spin-orbit interaction in the process of ultrafast laser-induced demagnetization is beyond the scope of this manuscript and will be an intriguing subject for future studies.

\section{CONCLUSIONS}

Ultrafast laser-induced magnetization dynamics in rareearth transition-metal alloys is investigated by both the magneto-optical Kerr effect and $\mathrm{THz}$ emission spectroscopy. Using the Maxwell equations we developed a framework allowing a comparative analysis of these two techniques. Application of these methods to pure Co and GdFeCo gives a good qualitative and quantitative agreement between the

TABLE I. Full width at half maximum (FWHM) and expectation value (i.e., center of mass) of the spectra presented in Fig. 4(b).

\begin{tabular}{llll}
\hline \hline & \multicolumn{1}{c}{ Co } & GdFeCo & NdFeCo \\
\hline FWHM $_{\mathrm{MOKE}}$ & $0.62 \mathrm{THz}$ & $0.76 \mathrm{THz}$ & $0.70 \mathrm{THz}$ \\
FWHM $_{\mathrm{THz}}$ & $0.58 \mathrm{THz}$ & $0.73 \mathrm{THz}$ & $0.81 \mathrm{THz}$ \\
$\left|\mathrm{FWHM}_{\mathrm{THz}}-\mathrm{FWHM}_{\mathrm{MOKE}}\right| / \mathrm{FWHM}_{\mathrm{MOKE}}$ & $6.1 \%$ & $4.4 \%$ & $0.76 \mathrm{THz}$ \\
mean $_{\mathrm{MOKE}}$ & $0.65 \mathrm{THz}$ & $0.79 \mathrm{THz}$ & $0.67 \mathrm{THz}$ \\
mean $_{\mathrm{THz}}$ mean $_{\mathrm{THz}}-$ mean $_{\mathrm{MOKE}} /$ mean $_{\mathrm{MOKE}}$ & $0.64 \mathrm{THz}$ & $4.2 \%$ & $0.77 \mathrm{THz}$ \\
\hline \hline
\end{tabular}


results provided by the magneto-optical Kerr effect and $\mathrm{THz}$ emission spectroscopy. For $\mathrm{NdFeCo}$ the $\mathrm{THz}$ emission indicates faster magnetization dynamics than that deduced from the magneto-optical response.

\section{ACKNOWLEDGMENTS}

We would like to thank T. Toonen and A. van Etteger for technical support. We would like to thank J. D. Costa for providing the Co sample, M. Huijben for the vibrating magnetometer measurements, and J. Becker for fruitful discussions. This work was supported by the Foundation for Fundamental Research on Matter (FOM), the European Unions Seventh Framework Program (Program No. FP7/2007-2013) Grant No. 280555 (Go-Fast), European Research Council Grant No. 257280 (Femtomagnetism), and the program "Leading Scientist" of the Russian Ministry of Education and Science (Grant No. 14.Z50.31.0034).

\section{APPENDIX}

Spectral dependence of the conductivity in the $\mathrm{THz}$ frequency range may modify the $\mathrm{THz}$ spectra significantly. To verify if such a spectral dependence plays a significant role in our measurements, we applied the conventional method of $\mathrm{THz}$ transmission time-domain spectroscopy. Moreover, we derived an expression for the transmission of $\mathrm{THz}$ radiation through a metal film as shown in Fig. 3(a). In the derivation we neglect any magnetization dynamics. In this case the derivative of the electric fields at the interfaces is continuous. The resulting expression for the transmission coefficient is

$$
t=\frac{4 k k_{0}}{\left(k+k_{0}\right)\left(k+k_{s}\right) e^{-i k d}-\left(k-k_{0}\right)\left(k-k_{s}\right) e^{i k d}} .
$$

Again we can use a Taylor expansion of the form $e^{ \pm i k d} \approx$ $1 \pm i k d$ together with the assumption of $k^{2} \gg k_{0} k_{s}$ to get

$$
t \approx \frac{2}{(1+n)+4 \pi \sigma d / c}
$$

which is consistent with the literature [33,34]. We can see that Eqs. (4) and (A1) [or (5) and (A2)] only differ by the nominator. Therefore, additional information from $\mathrm{THz}$ transmission can help us understand $\mathrm{THz}$ emission better.

If we are not in the thin layer limit $[(1+n) \gg 4 \pi \sigma d / c]$, then the discrepancy in spectral bandwidth observed between MOKE and $\mathrm{THz}$ emission of $\mathrm{NdFeCo}$ may be explained by $\mathrm{NdFeCo}$ having a conductivity which changes rapidly upon an increase of the frequency. Since the $\mathrm{THz}$ emission from $\mathrm{GdFeCo}$ is well described in the thin layer limit, we can use it as a reference. For this reason we performed additional THz transmission measurements of the NdFeCo and GdFeCo samples. A rapid change of the conductivity should originate from spectral dependence of the denominator in Eq. (A2). This denominator is similar to the one in Eq. (5). Hence only a large difference in $\mathrm{THz}$ transmission of $\mathrm{NdFeCo}$ and $\mathrm{GdFeCo}$ samples can explain the discrepancy in spectral bandwidth of $\mathrm{NdFeCo}$. In Fig. 5(a) we show the transmission of $\mathrm{Nd}_{0.2}\left(\mathrm{Fe}_{0.87} \mathrm{Co}_{0.13}\right)_{0.8}$ normalized to the transmission of $\mathrm{Gd}_{0.3}\left(\mathrm{Fe}_{0.87} \mathrm{Co}_{0.13}\right)_{0.7}$. It is apparent that below $1 \mathrm{THz}$ there is an obvious difference in transmission which may indicate the presence of a frequencydependent conductivity contribution. However, when using this transmission ratio to determine a possible correction to the emission spectrum of $\mathrm{NdFeCo}$ [see Fig. 5(b)], there is still a pronounced mismatch between the measured and calculated spectra. This fact excludes the possibility that for one type of samples the thin layer limit is reasonable and not for the other.
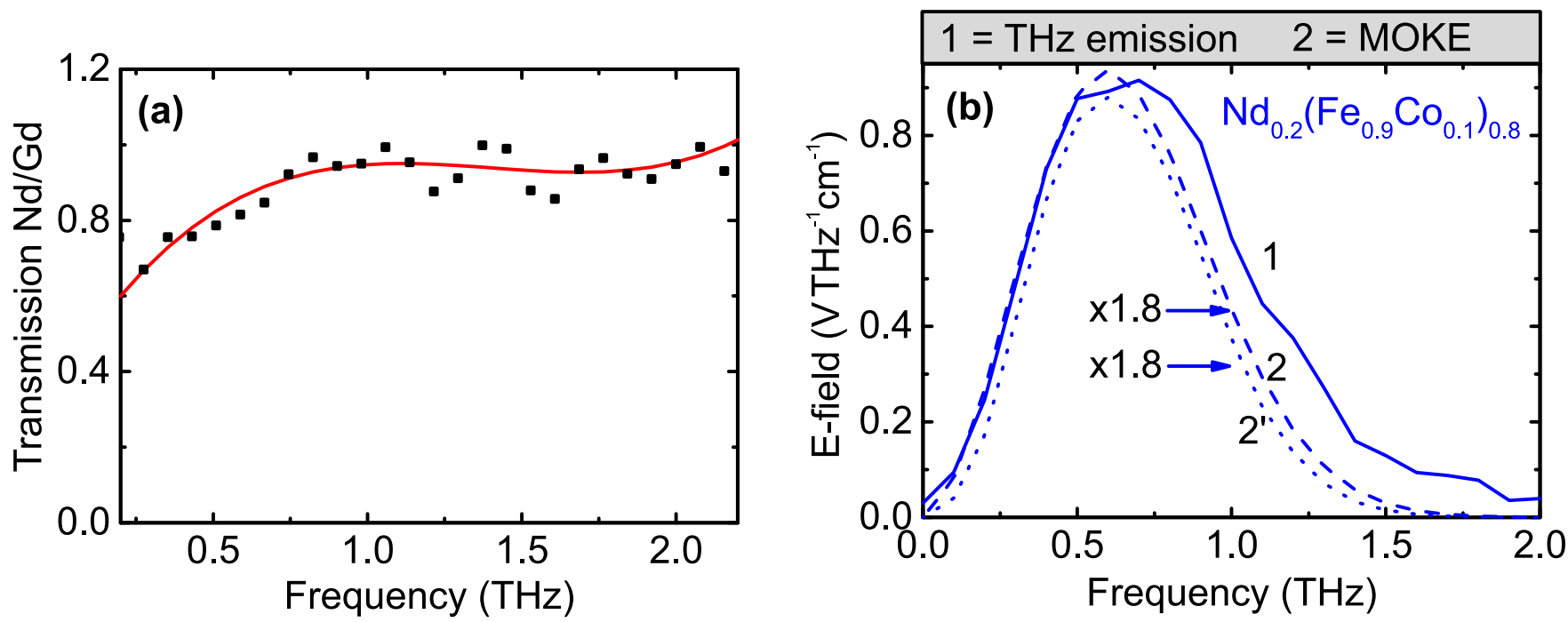

FIG. 5. (Color online) (a) The transmission of $\mathrm{Nd}_{0.2}\left(\mathrm{Fe}_{0.87} \mathrm{Co}_{0.13}\right)_{0.8}$ normalized to the transmission of $\mathrm{Gd}_{0.3}\left(\mathrm{Fe}_{0.87} \mathrm{Co}_{0.13}\right)_{0.7}$. The solid line is a polynomial fit. (b) The solid line (1) is the spectrum obtained with the help of the Fourier transform of the measured THz emission from $\mathrm{Nd}_{0.2}\left(\mathrm{Fe}_{0.87} \mathrm{Co}_{0.13}\right)_{0.8}$. The dashed line (2) indicates the calculated spectrum from the MOKE and taking into account the spectrometer response for $\mathrm{THz}$ radiation. The dotted line $\left(2^{\prime}\right)$ indicates a possible correction to the calculated spectrum using the transmission data of (a). The calculated spectra are shown multiplied by 1.8 . 
[1] E. Beaurepaire, J.-C. Merle, A. Daunois, and J.-Y. Bigot, Ultrafast Spin Dynamics in Ferromagnetic Nickel, Phys. Rev. Lett. 76, 4250 (1996).

[2] L. Guidoni, E. Beaurepaire, and J.-Y. Bigot, Magneto-optics in the Ultrafast Regime: Thermalization of Spin Populations in Ferromagnetic Films, Phys. Rev. Lett. 89, 017401 (2002).

[3] J.-Y. Bigot, M. Vomir, and E. Beaurepaire, Coherent ultrafast magnetism induced by femtosecond laser pulses, Nat. Phys. 5, 515 (2009).

[4] G. P. Zhang, W. Hübner, G. Lefkidis, Y. Bai, and T. F. George, Paradigm of the time-resolved magneto-optical Kerr effect for femtosecond magnetism, Nat. Phys. 5, 499 (2009).

[5] A. Kirilyuk, A. V. Kimel, and Th. Rasing, Ultrafast optical manipulation of magnetic order, Rev. Mod. Phys. 82, 2731 (2010).

[6] B. Koopmans, G. Malinowski, F. Dalla Longa, D. Steiauf, M. Fähnle, T. Roth, M. Cinchetti, and M. Aeschlimann, Explaining the paradoxical diversity of ultrafast laser-induced demagnetization, Nat. Mater. 9, 259 (2010).

[7] M. Battiato, K. Carva, and P. M. Oppeneer, Superdiffusive Spin Transport as a Mechanism of Ultrafast Demagnetization, Phys. Rev. Lett. 105, 027203 (2010).

[8] K. Carva, M. Battiato, and P. M. Oppeneer, Is the controversy over femtosecond magneto-optics really solved? Nat. Phys. 7, 665 (2011).

[9] H. Regensburger, R. Vollmer, and J. Kirschner, Time-resolved magnetization-induced second-harmonic generation from the Ni(110) surface, Phys. Rev. B 61, 14716 (2000).

[10] B. Koopmans, M. van Kampen, J. T. Kohlhepp, and W. J. M. de Jonge, Ultrafast Magneto-Optics in Nickel: Magnetism or Optics? Phys. Rev. Lett. 85, 844, (2000).

[11] A. K. Zvezdin and V. A. Kotov, Modern Magnetooptics and Magnetooptical Materials, 1st ed. (Institute of Physics Publishing, Bristol and Philadelphia, 1997).

[12] C. Boeglin, E. Beaurepaire, V. Halté, V. López-Flores, C. Stamm, N. Pontius, H. A. Dürr, and J.-Y. Bigot, Distinguishing the ultrafast dynamics of spin and orbital moments in solids, Nature 465, 458 (2010).

[13] I. Radu, K. Vahaplar, C. Stamm, T. Kachel, N. Pontius, H. A. Dürr, T. A. Ostler, J. Barker, R. F. L. Evans, R. W. Chantrell, A. Tsukamoto, A. Itoh, A. Kirilyuk, Th. Rasing, and A. V. Kimel, Transient ferromagnetic-like state mediating ultrafast reversal of antiferromagnetically coupled spins, Nature 472, 205 (2011).

[14] A. R. Khorsand, M. Savoini, A. Kirilyuk, A. V. Kimel, A. Tsukamoto, A. Itoh, and Th. Rasing, Element-Specific Probing of Ultrafast Spin Dynamics in Multisublattic Magnets with Visible Light, Phys. Rev. Lett. 110, 107205 (2013).

[15] C. E. Graves et al., Nanoscale spin reversal by non-local angular momentum transfer following ultrafast laser excitation in ferrimagnetic GdFeCo, Nat. Mater. 12, 293 (2013).

[16] N. Bergeard, V. López-Flores, V. Halté, M. Hehn, C. Stamm, N. Pontius, E. Beaurepaire, and C. Boeglin, Ultrafast angular momentum transfer in multisublattice ferrimagnets, Nat. Commun. 5, 3466 (2014).

[17] E. Beaurepaire, G. M. Turner, S. M. Harrel, M. C. Beard, J.-Y. Bigot, and C. A. Schmuttenmaer, Coherent terahertz emission from ferromagnetic films excited by femtosecond laser pulses, Appl. Phys. Lett. 84, 3465 (2004).
[18] D. J. Hilton, R. D. Averitt, C. A. Meserole, G. L. Fisher, D. J. Funk, J. D. Thompson, and A. J. Taylor, Terahertz emission via ultrashort-pulse excitation of magnetic metal films, Opt. Lett. 29, 1805 (2004).

[19] J. Shen, H.-W. Zhang, and Y.-X. Li, Terahertz emission of ferromagnetic Ni-Fe thin films excited by ultrafast laser pulses, Chin. Phys. Lett. 29, 067502 (2012).

[20] T. Kampfrath, M. Battiato, P. Maldonado, G. Eilers, J. Nötzold, S. Mährlein, V. Zbarsky, F. Freimuth, Y. Mokrousov, S. Blügel, M. Wolf, I. Radu, P. M. Oppeneer, and M. Münzenberg, Terahertz spin current pulses controlled by magnetic heterostructures, Nat. Nanotechnol. 8, 256 (2013).

[21] J. N. Heyman, P. Neocleous, D. Hebert, P. A. Crowell, T. Müller, and K. Unterrainer, Terahertz emission from GaAs and InAs in a magnetic field, Phys. Rev. B 64, 085202 (2001).

[22] M. A. Ordal, R. J. Bell, R. W. Alexander, L. L. Long, and M. R. Querry, Optical properties of fourteen metals in the infrared and far infrared: $\mathrm{Al}, \mathrm{Co}, \mathrm{Cu}, \mathrm{Au}, \mathrm{Fe}, \mathrm{Pb}, \mathrm{Mo}, \mathrm{Ni}, \mathrm{Pd}$, Pt, Ag, Ti, V, and W, Appl. Opt. 24, 4493 (1985).

[23] L. Duvillaret, F. Garet, and J.-L. Coutaz, A reliable method for extraction of material parameters in terahertz time-domain spectroscopy, IEEE J. Sel. Top. Quantum Electron. 2, 739 (1996).

[24] M. Naftaly and R. E. Miles, Terahertz time-domain spectroscopy of silicate glasses and the relationship to material properties, J. Appl. Phys. 102, 043517 (2007).

[25] P. Kužel, M. A. Khazan, and J. Kroupa, Spatiotemporal transformations of ultrashort terahertz pulses, J. Opt. Soc. Am. B 16, 1795 (1999).

[26] G. Gallot, J. Zhang, R. W. McGowan, T.-I. Jeon, and D. Grischkowsky, Measurements of the $\mathrm{THz}$ absorption and dispersion of ZnTe and their relevance to the electro-optic detection of THz radiation, Appl. Phys. Lett. 74, 3450 (1999).

[27] P. C. M. Planken, H.-K. Nienhuys, H. J. Bakker, and T. Wenckebach, Measurement and calculation of the orientation dependence of terahertz pulse detection in ZnTe, J. Opt. Soc. Am. B 18, 313 (2001).

[28] Magnetic Interactions and Spin Transport, edited by A. Chtchelkanova, S. A. Wolf, and Y. Idzerda (Springer, New York, 2003).

[29] M. Ding and S. J. Poon, Tunable perpendicular magnetic anisotropy in GdFeCo amorphous films, J. Magn. Magn. Mater. 339, 51 (2013).

[30] R. C. Taylor, T. R. McGuire, J. M. D. Coey, and A. Gangulee, Magnetic properties of amorphous neodymium-transitionmetal films, J. Appl. Phys. 49, 2885 (1978).

[31] R. J. Gambino and T. R. McGuire, Magnetooptic properties of NdFeCo amorphous alloys, J. Appl. Phys. 57, 3906 (1985).

[32] J.-Y. Kim, J.-H. Kim, and H.-W. Oh, Comparisons of magnetic and magneto-optic properties between Fe-rich and Nd-rich amorphous $\mathrm{Nd}_{x} \mathrm{Fe}_{1-x}$ alloys, J. Magn. 3, 49 (1998).

[33] M. Tinkham, Energy gap interpretation of experiments on infrared transmission through superconducting films, Phys. Rev. 104, 845 (1956).

[34] M. Walther, D. G. Cooke, C. Sherstan, M. Hajar, M. R. Freeman, and F. A. Hegmann, Terahertz conductivity of thin gold films at the metal-insulator percolation transition, Phys. Rev. B 76, 125408 (2007). 